CT-FFR with computerised tomography coronary angiography (CTCA) against the gold standard of ICA in different realworld patient groups.

Methods We conducted a retrospective study of 477 patients referred for CTCA or CT-FFR for investigation of possible coronary ischaemia. Patients were excluded if the image quality was poor or inconclusive. Patient-based PPV was calculated to detect or rule out significant $\mathrm{CAD}$, defined as more than $70 \%$ stenosis on ICA. A sub-analysis of PPV by indication for scan was also performed. Patients that underwent invasive non-hyperaemic pressure wire measurements had their iFR or RFR compared with their CT-FFR values.

Results In a patient-based analysis, the overall PPV was 59.3\% for CTCA and $76.2 \%$ for CT-FFR. This increased to $81.0 \%$ and $86.7 \%$ respectively for patients with stable angina symptoms. In patients with atypical angina symptoms, CT-FFR considerably outperformed CTCA with a PPV of $61.3 \%$ vs. $37.5 \%$. There was not a linear relationship between invasive pressure wire measurement and CT-FFR value $(r=0.23$, $\mathrm{p}=0.265$ ).

Conclusion The PPV of CTCA and CT-FFR is lower in the real-world than in previously published trials, partly due to the heterogeneity of indication for the scan. However, in patients with typical angina symptoms, both are reliable diagnostic tools to determine the presence of clinically significant coronary stenoses. CT-FFR significantly outperforms CTCA in patients with more atypical symptoms and the targeted use of CT-FFR in this group may help to avoid unnecessary invasive procedures.

Conflict of Interest None

\section{WOMEN WITH EARLY ONSET HYPERTENSION ARE MORE LIKELY TO HAVE CARDIAC MRI FINDINGS ASSOCIATED WITH FUTURE DEVELOPMENT OF HEART FAILURE THAN MEN}

${ }^{1}$ Howell Williams, ${ }^{2}$ Jonathan Rodrigues, ${ }^{3}$ Konstantina Mitrousi, ${ }^{4}$ Will Ormerod ${ }^{4}$ Dylan Walker, ${ }^{5}$ Nathan Manghat, ${ }^{6}$ Emma Hart, ${ }^{3}$ Angus Nightingale. ${ }^{7}$ North Bristol NHS Trust, Bristol, UK; ${ }^{2}$ Department of Radiology, Royal United Hospitals Bath, UK; ${ }^{3}$ Department of Cardiology, Bristol Heart Institute, UK; ${ }^{4}$ Bristol Medical School, University of Bristol, UK; ${ }^{5}$ Department of Radiology, Bristol Heart Institute, UK; ${ }^{6}$ School of Physiology, Pharmacology and Neuroscience, University of Bristol, UK

\subsection{6/heartjpl-2021-BCS.164}

Introduction Hypertension (HTN) is a major risk factor for developing of heart failure (HF). Patients with early onset HTN ( $\leq 40$ years) are at increased risk compared to those who develop HTN in later life. The transition from HTN to $\mathrm{HF}$ is incompletely understood but involves left ventricular (LV) changes at the cellular level. These include diffuse interstitial fibrosis, measured by extracellular volume fraction (ECV). Elevated ECV is associated with an increased risk of death and HF hospitalisation but the extent of elevated ECV in patients with early onset HTN is not known. In addition, among patients with $\mathrm{HF}$ with preserved ejection fraction (HFpEF), women outnumber men by a ratio of $2: 1$. The reasons for this are not known.

We aimed to investigate cardiac MRI parameters including ECV in patients with early onset HTN who do not have HF. Methods Data from consecutive patients from a tertiary HTN clinic referred for CMR (Siemens, Germany) over a 3-year period was retrospectively analysed. Elevated ECV was defined
Abstract 167 Table 1 Factors assessed for differences between patients $\leq 40$ years with $\mathrm{ECV} \geq 28 \%$ vs $\mathrm{ECV}<28 \%$

\begin{tabular}{|l|c|c|c|}
\hline \multicolumn{1}{|c|}{ Parameter } & ECV $<\mathbf{2 8 \%}$ & $\mathbf{E C V ~} \geq \mathbf{2 8 \%}$ & P value (test) \\
\hline Age $($ years $)$ & $33.3+/-5.7$ & $33.1+/-5.1$ & 0.93 (t-test) \\
\hline BMI $\left(\mathrm{kg} / \mathrm{m}^{2}\right)$ & $30.8+/-6.9$ & $30.4+/-7.7$ & 0.90 (t-test) \\
\hline Office $\mathrm{SBP}(\mathrm{mmHg})$ & $151+/-16$ & $151+/-24$ & 0.92 (t-test) \\
\hline Office DBP $(\mathrm{mmHg})$ & $95+/-10$ & $95+/-9$ & 0.97 (t-test) \\
\hline Number of antihypertensives taken & $1.7+/-1.6$ & $1.7+/-1.8$ & $0.98($ t-test) \\
\hline Treatment with ACE-inhibitor or ARB & $13 / 20$ & $6 / 9$ & 1.0 (Fisher's exact) \\
\hline
\end{tabular}

as $\geq 28 \%$ as this is associated with increased HF hospitalisation. Data are mean \pm SD. Alpha was set at 0.05 .

Results 29 patients $\leq 40$ years underwent CMR during the study period; mean age $33 \pm 5.4$ years, 15/29 (52\%) female. Elevated ECV was identified in $9 / 29$ (31\%) patients $\leq 40$ years and the majority were female $(8 / 9, p=0.01$, Fisher's exact test). This gender difference was not seen in patients $>40$ years old with HTN who underwent CMR during the same period (29/59 [49\%] had an elevated ECV with 14/29 female, $p=0.19$, Fisher's exact test).Among patients $\leq 40$ years, LV hypertrophy (all concentric) was more common in those with ECV $\geq 28 \%$ (5/9) than those with ECV $<28 \%$ (2/ $20, p=0.02$, Fisher's exact test). Among the female patients $\leq 40$ years, there was a trend towards increased LV mass in the 8 patients with ECV $\geq 28 \%$ compared to 7 with ECV $<28 \%(78 \pm 14.4 \mathrm{~g} / \mathrm{m} 2$ vs $67 \pm 3.8 \mathrm{~g} / \mathrm{m} 2, \mathrm{p}=0.06$, t-test).Among patients $\leq 40$ years, there were no significant differences between the ECV $\geq 28 \%$ and ECV $<28 \%$ groups in LV ejection fraction or LV strain, strain rate, and diastolic strain rate. There were also no differences in the parameters in table 1.

Conclusions Among patients with early onset HTN, one in three had elevated ECV. This was independent of age, BMI, blood pressure (BP), and BP treatment. These patients are likely to be at increased risk of $\mathrm{HF}$ in later life.Patients with early onset HTN and an elevated ECV were more likely to be female. This may reflect an expected gender difference; recent studies indicate healthy females have higher ECV than healthy males, and this study further highlights the importance of separate ECV reference ranges for men and women. However it may also reflect early pathological fibrosis in response to HTN, particularly as in patients $>40$ years old there was no gender difference among patients with an elevated ECV. Increased LV fibrosis at a younger age may be a contributor to the increased development of HFpEF among female patients.

Conflict of Interest None

\section{PREVALENCE AND ADVERSE CARDIAC EVENTS IN ANOMALOUS AORTIC ORIGIN OF CORONARY ARTERY (AAOCA) IN CT CORONARY ANGIOGRAPHY IN WEST OF SCOTLAND}

${ }^{1}$ Punit Bedi, ${ }^{2}$ Oliver Peck, ${ }^{2}$ Jacqueline Adams. ${ }^{1}$ Royal Alexandra Hospital, Glasgow, UK; ${ }^{2}$ Golden Jubilee National Hospital Glasgow

\subsection{6/heartjnl-2021-BCS.165}

Background Anomalous aortic origin of a coronary artery (AAOCA) is a congenital abnormality of the origin or course of coronary artery. The clinical manifestations of coronary artery anomalies vary in severity based on its course. It is reported as a leading cause of sudden cardiac death in young 